Textures and Microstructures, 1988, Vol. 10, pp. 9-19

Reprints available directly from the publisher

Photocopying permitted by license only

(C) 1988 Gordon and Breach Science Publishers Inc.

Printed in the United Kingdom

\title{
Microstructure and Texture of Rolled (110)[001] Copper Single Crystals
}

\author{
M. WRÓBEL, S. DYMEK, M. BLICHARSKI and \\ S. GORCZYCA
}

Department of Metallurgy, Academy of Mining and Metallurgy, Kraków Poland

(Received November 11, 1987)

The dislocation structure was free from inhomogeneities with high orientation changes normally observed in deformed materials. After reductions $20 \%$ and $40 \%$ against the background of cells there were sometimes observed bands (dislocation rich layers). With increasing strain the cell structure became faint and the dislocation rich layers became sharper (after 60\% reduction no dislocation cells were revealed). It has been found, that in layers lying close to the rolled surface of the specimen the dislocation bands were nearly parallel to the RD and much thinner than those in further layers. SAD analysis indicate that in the surface layers the crystallographic orientation changed systematically with increasing distance from the surface to the center of the specimen (rotation around ND). In the intermediate layers the dislocation bands were curved and overlapped the other dislocation bands. Despite of detailed investigation shear bands were not observed even in specimens rolled to the $95 \%$ reduction.

KEY WORDS: Copper, Single crystal, Rolling deformation, Pole figures, Electron microscopy, Selected area diffraction.

\section{INTRODUCTION}

It is commonly accepted that (110)[001] f.c.c. single crystals are metastable during deformation by rolling. It has been proved using the Sachs model, that under the condition of plane stress state this orientation is stable in relation to the rotation around rolling direction (RD) and transverse direction (TD). However, it is unstable in relation to each, even very slight fluctuation which occur around normal direction (ND) and causes rotation toward two 


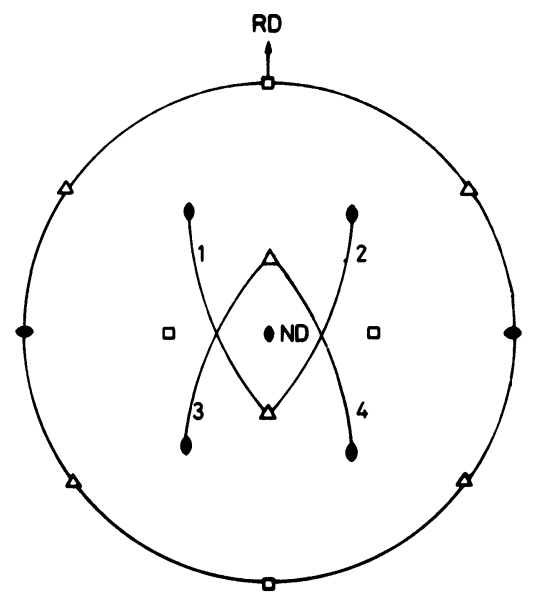

Figure 1 Slip systems operating in the (110)[001] crystal.

symmetric stable end-orientation $\{110\}\langle 112\rangle$ (Aernouldt et al. 1979).

On the other hand, according to classical Taylor's theory under conditions of plane strain state the orientation (110)[001] does not alter (Dilamore et al. 1968). It has been calculated recently that during rolling of f.c.c. single crystals being deformed by slip in $\{111\}\langle 110\rangle$ systems the orientation (110)[001] can change by rotation around ND or TD or it can remain stable (Latas et al. 1987). This depends on the constraints imposed on the strain field and on the assumed relation between strain and critical stresses in active slip systems and is a consequence of a shear strain change in the active slip systems $n r 1,2,3,4$ (Figure 1).

The texture investigation of (110)[001] oriented single crystals deformed by homogeneous rolling proving that up to $90 \%$ reduction the crystal retained its orientation only in the centre layer of the rolled specimen (Bauer et al. 1977). In the intermediate layers the initial orientation is smeared due to the rotation around TD. In the surface layers the spread of the initial orientation exists after $80 \%$ reduction due to the rotation around ND.

The aim of the present research is to explain the observed changes of texture on the basis of the dislocation microstructure 
which developed in rolled copper single crystals with orientation (110)[001].

\section{EXPERIMENTAL PROCEDURE}

Single crystals of the size $8 \times 30 \times 100 \mathrm{~mm}$ were rolled in both directions by reversing end to end between passes up to a reduction of $95 \%$. The ratio $l / h$ characterizing the geometry of the rolled gap was kept between 1.1 and 3 during each pass ( $l$-the projected length of contact between the roll and the material, $h$-mean thickness of the sample). Specimens for investigations were cut from the rolled bar at various degrees of rolling. During the examination of the microstructure by TEM the distance of the examined region from the rolling surface was determined.

\section{RESULTS}

The texture results for the centre layer of the rolled copper single crystals (Figure 2) were very similar to those obtained by Bauer et al. (1977) for the same orientation.

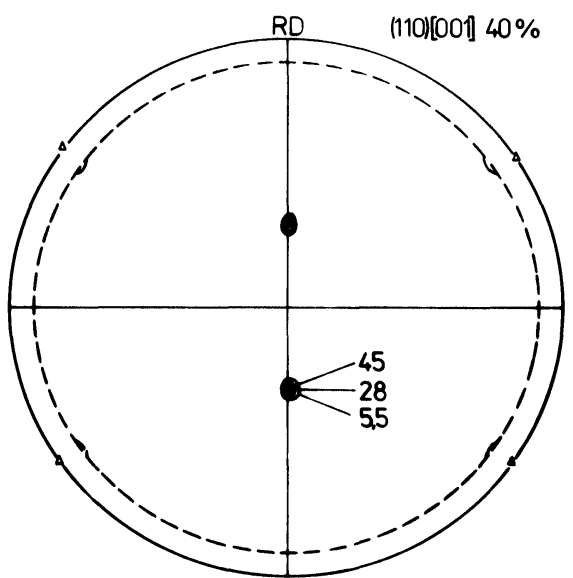

(a)

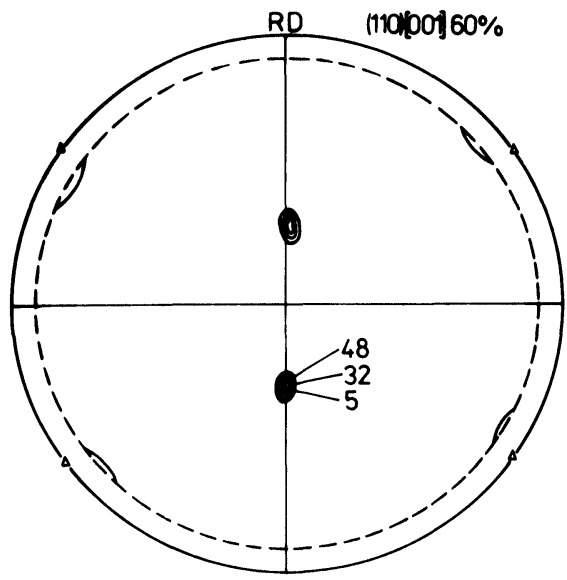

(b)

Figure 2 Texture in the central layer of a crystal with initial orientation (110)[001]; deformation (a) $40 \%$, (b) $60 \%$. 


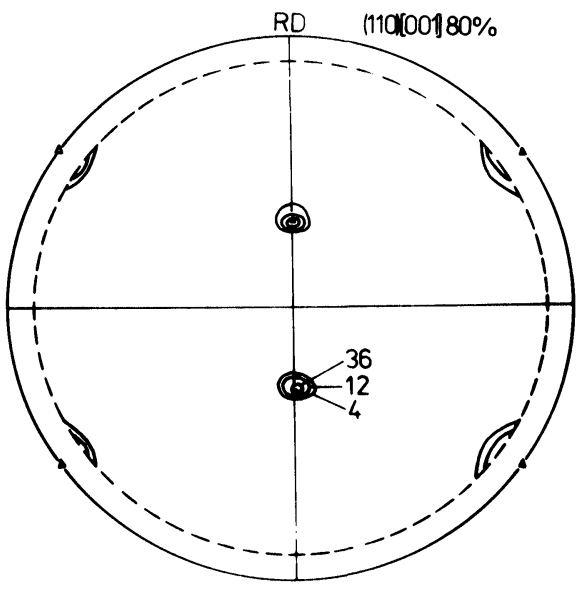

(c)

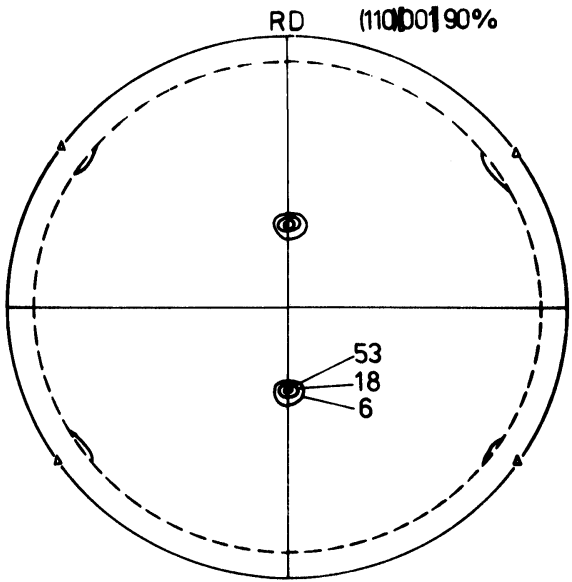

(d)

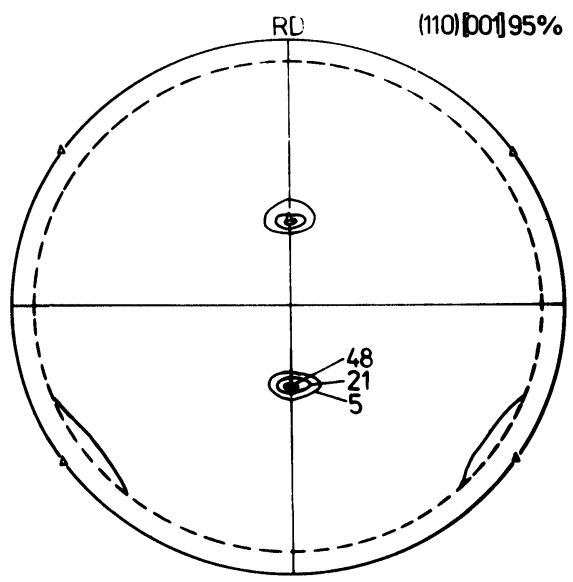

(e)

Figure 2 (Continued) Texture in the central layer of a crystal with initial orientation (110)[001]; deformation (c) $80 \%$, (d) $90 \%$, (e) $95 \%$.

The metallographic specimens show uniform etching throughout the thickness of the samples after each given amount of deformation. The etchant used revealed slip plane traces the distribution of which were uniform. These traces intersected at an angle of $70^{\circ}$ and lay symmetrically with respect to the RD (Figure 3).

The dislocation structure was relatively homogeneous without the high orientation gradients normally observed in deformed materials 


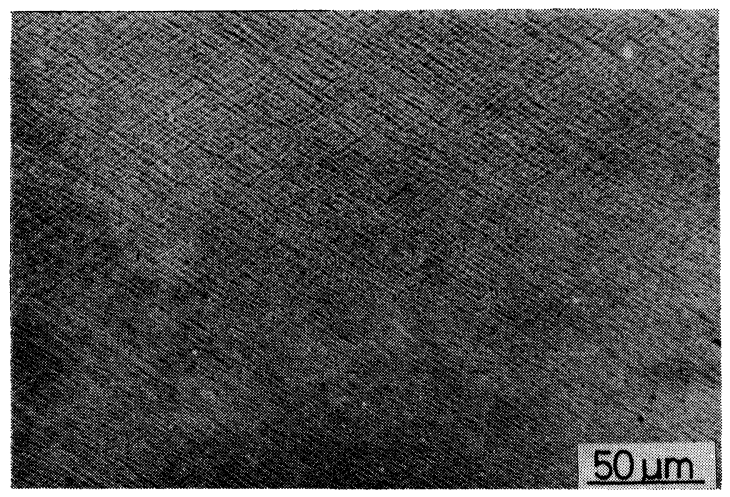

Figure 3 Slip plane traces; $40 \%$ deformation; the longer edge is parallel to the rolling direction.

even at low amounts of deformation. After $20 \%$ reduction the microstructures of the present crystal consisted of faintly shaped irregular cells the diameter of which changed from 0.15 to $1.5 \mu \mathrm{m}$. Against the background of the dislocation cell structure dislocation bands (dislocation rich layers were occasionally observed) which were inclined at an angle of $\pm 35^{\circ}$ to the RD (Figure 4). After small deformation the dislocation bands were waved and their thickness was not constant. With increasing strain the cell structure became faint and the dislocation rich layers became sharper (after $60 \%$ reduction no dislocation cells were revealed). However, the two directional dislocation bands intersecting or interpenetrating each other were observed. In some regions only one set of dislocation bands was observed caused by satisfying the contrast condition on the electron image. When the thin foil was slightly tilted the second set of dislocation bands became visible (Figure 5). The dislocation rich layers became thinner and thinner and more straight with increasing deformation and the regions between them contained fewer and fewer dislocations. Occasionally at the place of the dislocation rich layers intersecting the segments of the dislocation bands were approximately parallel to the RD (Figure 6). The volume fraction of such segments increased with increasing deformation but bands inclined at an angle of $\pm 35^{\circ}$ to the RD were also observed. An important feature of the examined microstructure was its relatively significant uniformity in large areas (Figure 7). 

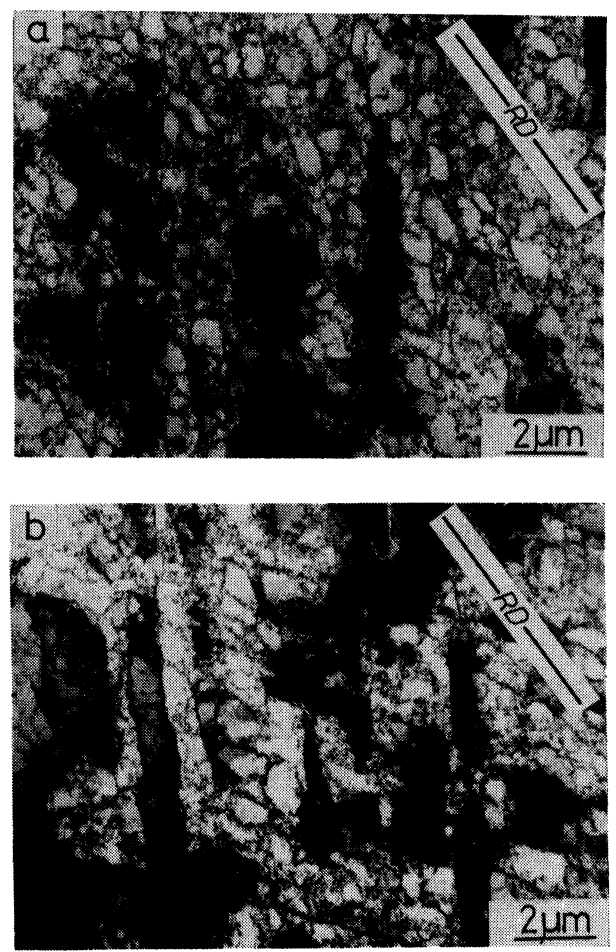

Figure 4 Cell structure and first dislocation rich layers; $20 \%$ deformation.

The dislocation microstructure after higher degrees of deformation was examined in various layers of the specimen. It was found, that in layers lying close to the rolled surface of the sample the dislocation bands were nearly parallel to the RD and much thinner than those in further layers (Figure 8). The crystallographic orientation changed systematically with increasing distance from the surface to the centre of the specimen. The SAD analysis proved that near the rolled surface the crystallographic lattice rotated around ND and the rotation decreased with the distance from the surface of the specimen. In the intermediate layers the dislocation bands were curved and overlapped the other dislocation bands (Figure 9). 

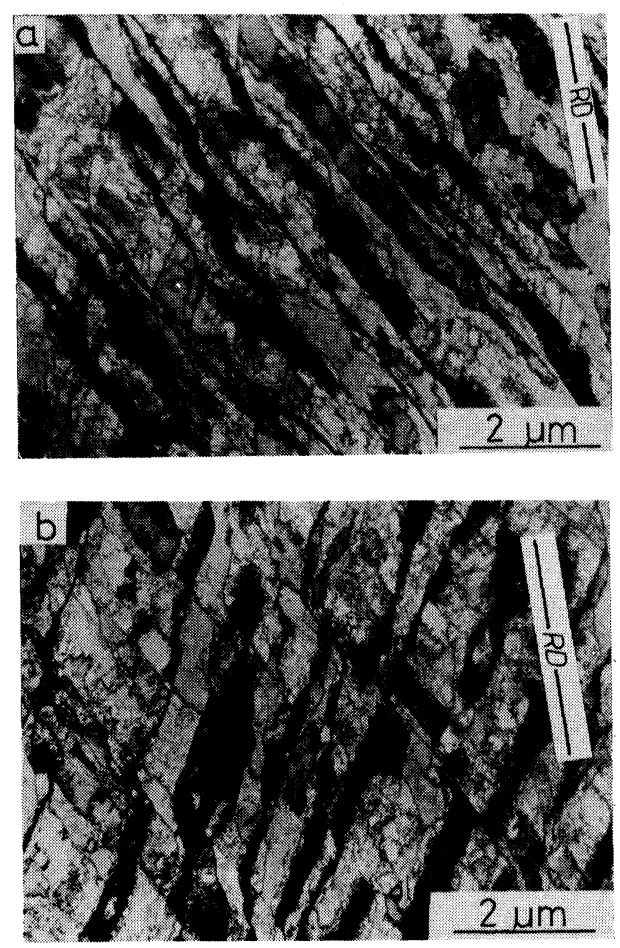

Figure 5 Two sets of dislocation bands in the same region. Micrographs (a) and (b) represent the same area of a thin foil tilted at different angles around RD; $40 \%$ deformation.

In the regions containing such curved bands there was smearing of the orientation around the TD. In the centre layer of the specimen after $95 \%$ reduction the dislocation microstructure consisted of dislocation bands parallel to the RD. Even with detailed investigation shear bands were not observed.

\section{DISCUSSION}

The microstructure and texture development in the examined copper single crystals may be explained assuming that the deformation proceeds by slip in systems $1,2,3,4$ (four systems on two 


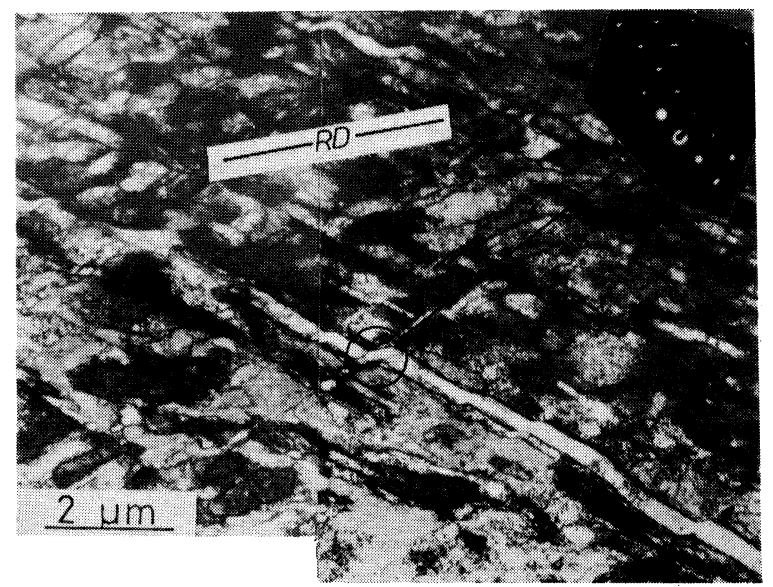

Figure 6 Intersection of dislocation rich layers; $60 \%$ deformation.
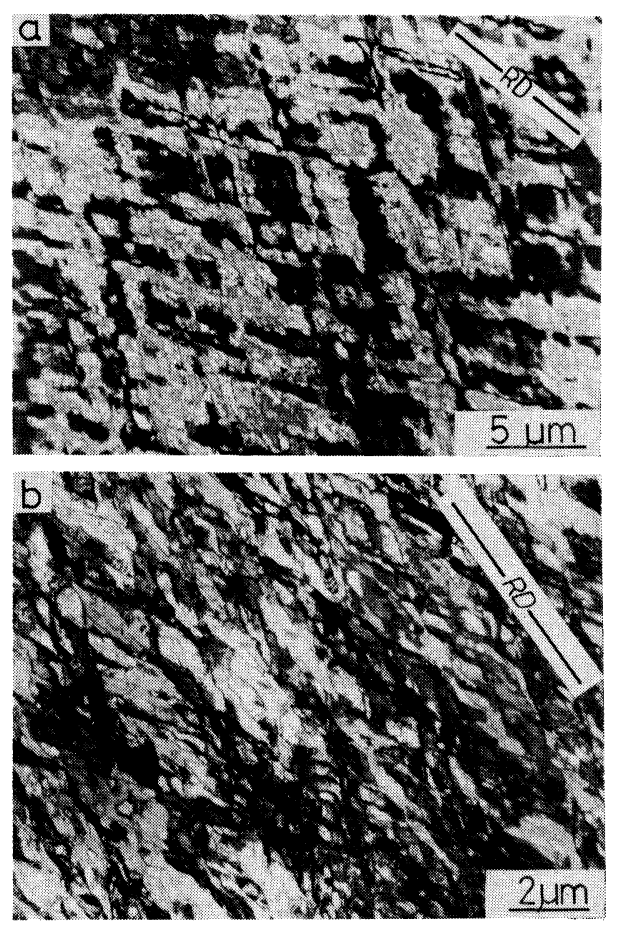

Figure 7 Deformation structure (a) 40\% deformation; (b) $80 \%$ deformation. 


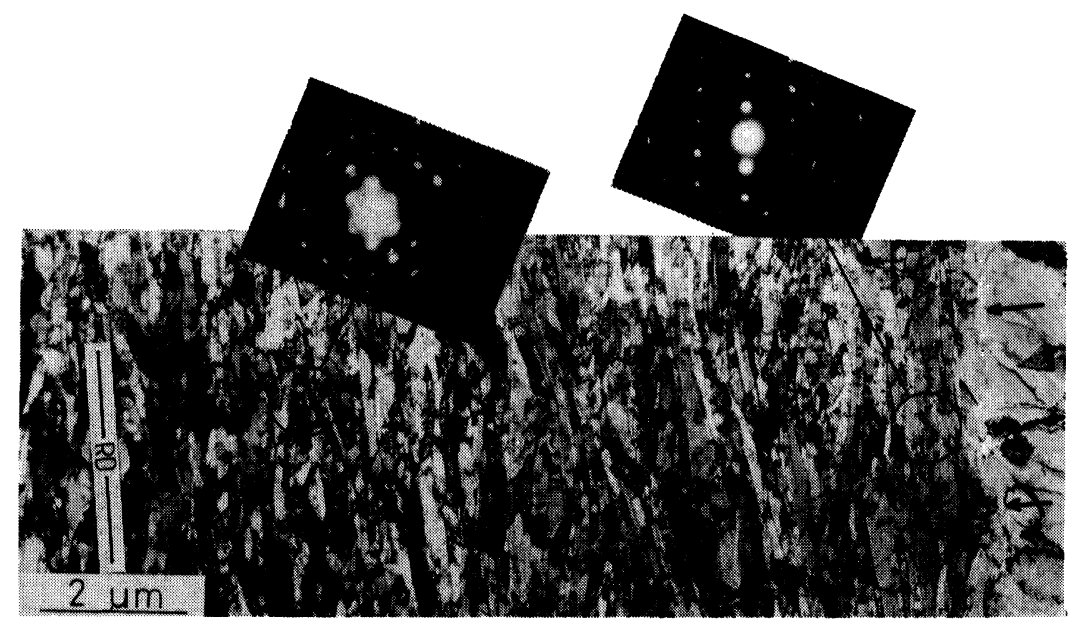

Figure 8 Dislocation rich layers close to the surface of the rolled crystal; $90 \%$ deformation. Arrows mark specimen surface.

planes)-Figure 1. Basing on the results of Latas et al. (1987), the smearing around TD in all layers of the sample observed by Bauer (1970) indicates that at a small degree of deformation slip occurs in all the mentioned systems and each system gives the same contribution to the total deformation. In the microstructure two sets of dislocation rich layers were observed, each set appearing as a result of dislocation accumulation on one set of the slip planes. The cell structure observed after low degree of deformation was probably due to the recovery of microstructure with low dislocation density. The origin of the cell structure formation could also be explained by the rearrangement of dislocations during thin foil preparation.

The changes of texture throughout the thickness of the specimen after heavy deformation (Bauer 1970) can be related to the changes of microstructure shown in this paper. The rotation of $\{111\}$ poles around ND in the surface and centre layers of the sample is caused by an absence of equilibrium of strains in the active slip systems. The shear strains in the slip systems 1 and 4 are not equal to those in systems 2 and 3 (Figure 1), but the total strains on both slip planes are equal. This probably caused the formation of dislocation rich layers parallel to the $\mathrm{RD}$ (Figure 8). Such a type of the 


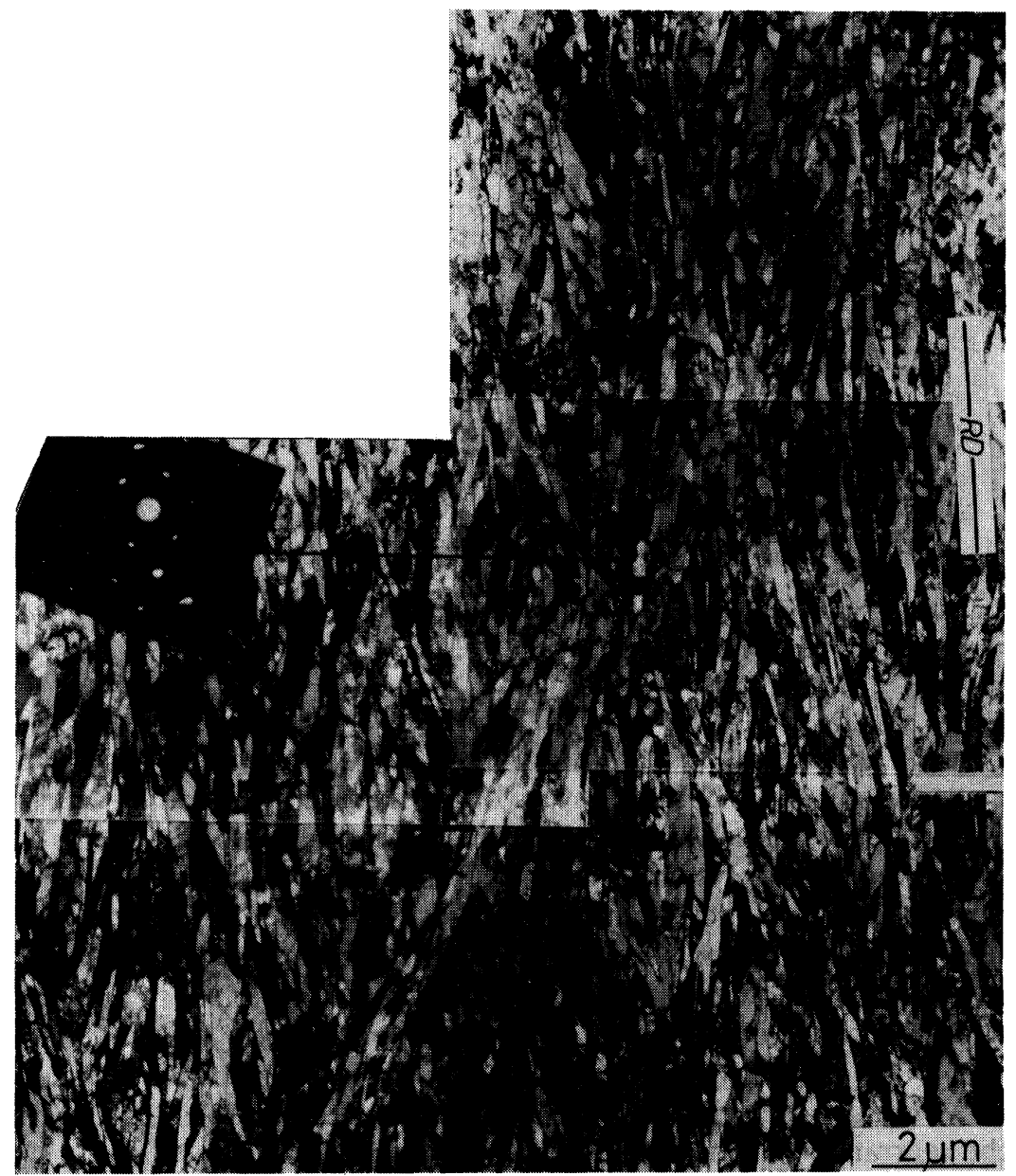

Figure 9 The banded structure in the intermediate layers; 95\% deformation.

microstructure dominates in the centre layer, in which the character of maximum spread on the pole figures also indicates for the rotation around ND.

The orientation smearing in the intermediate layers of the specimen revealed by Bauer (1970) may be explained by the overshooting phenomenon by the alternating deformation in both 
coplanar slip systems. Activation of the second pair of slip system is due to changes of the orientation parameter $M$. This occurs when higher hardening in the unactive pair of slip systems become balanced by the higher parameter $M$. The fluctuation of orientation around TD caused by momentarily unequal strains in both slip planes is additionally intensified by the oscillation of the stress state around TD. Such a deformation mode may explain the presence of curved dislocation bands in the intermediate layers. These curved dislocation bands may originate from the unequal shears on both slip planes which cause the smearing of the $\{111\}$ poles around TD observed both on pole figures by Bauer (1970) and on diffraction patterns in this paper (Figure 9).

\section{References}

Aernouldt, E. and Stüwe H. P. (1979) Z. Metallkunde 61, 128.

Bauer R. (1970) Ph.D. thesis, Aachen, West Germany.

Bauer, R., Mecking H. and Lücke K. (1977) Mat. Sc. Eng. 27, 162.

Dillamore, J. L., Butler, E. and Green D. (1968) Met. Sc. 2, 161.

Latas, W., Wróbel M. and Gorczyca S. (1987) Arch. of Metallurgy 32, 518. 